\title{
VULNERABILIDADE E RISCO EM UM ASSENTAMENTO URBANO NA PLANÍCIE DE INUNDAÇÃO DO RIO TIETÊ NO MUNICÍPIO DE SÃO PAULO (SP)
}

\author{
Camila Capassi MALAGODI \\ Alex Ubiratan Goossens PELOGGIA
}

\begin{abstract}
RESUMO
A expansão horizontal periférica dos grandes centros urbanos é caracterizada pela conjugação da ocupação de terrenos frágeis do ponto de vista geológico-geotécnico por contingentes populacionais econômico e socialmente desprivilegiados. O resultado comum dessa interação é o surgimento de áreas de risco, que se caracterizam por apresentarem gravidade e complexidade variável, e que exigem, por parte dos gestores públicos, medidas de caráter diferenciado, sendo necessária uma gestão de risco eficaz, subsidiada por ferramentas que auxiliem na tomada de decisões. Nesse contexto, este trabalho tem como objetivo determinar a vulnerabilidade social, o perigo e o risco associados à ocorrência de inundações no Jardim Pantanal, um assentamento urbano localizado na planície aluvial do rio Tietê no município de São Paulo, efetivamente atingido, e de forma recorrente, por tal fenômeno. O índice de vulnerabilidade social foi obtido pela soma de fatores relacionados ao bem-estar humano que integra os indivíduos expostos e afetados por um perigo, por meio de dados do censo demográfico de 2010, disponibilizados pelo IBGE por setor censitário. Os valores assumidos pelo perigo, ou aqui denominado nível de suscetibilidade à inundação $(N S)$, referem-se à cota topográfica predominante em cada setor da área, definidos pela elevação do terreno. Correlacionando-se tais índices a partir de técnicas de geoprocessamento, foi possível determinar e hierarquizar setores de maior ou menor risco de inundação. De maneira geral, os setores que apresentam os grupos mais frágeis são também os localizados mais próximos à várzea do rio, portanto em áreas altamente suscetíveis à ocorrência de inundações. Ao todo mais de 10 mil pessoas, sendo que mais da metade são mulheres e aproximadamente 2.500 são crianças, estão sob alto risco de ocorrência de novos desastres. A pobreza nesses setores também é um fator em destaque, o que intensifica a dificuldade ao acesso à água potável e saneamento, infraestrutura e abrigo seguro. Considera-se que a metodologia desenvolvida, aplicável a outras áreas em situações análogas, possa fornecer critérios de gestão de risco, notadamente no que tange ao planejamento e priorização de intervenções, e que ainda, em estudos futuros, devam ser realizados novos testes com outras variáveis.
\end{abstract}

Palavras-chave: Gestão de riscos; vulnerabilidade social; inundação; planície do rio Tietê; Jardim Pantanal.

\section{ABSTRACT}

VULNERABILITY AND RISK IN AN URBAN SETTLEMENT IN THE TIETE RIVER FLOODPLAIN IN THE SÃO PAULO MUNICIPALITY (SP). The peripheral expansion of great urban centers is characterized by the occupation of fragile areas in terms of geological and geotechnical parameters by an economically and socially disadvantaged population. The result of this interaction is the emergence of risk areas, which are very complex and present serious problems, requiring different responses from public authorities and an effective risk management relying on specific tools to assist in the decision-making process. In this context, this study aims to determine the social vulnerability, hazard and risk associated with flooding in Jardim Pantanal, an urban settlement frequently and effectively affected by flooding, located on the floodplain of 
the Tietê River, in the city of São Paulo. The social vulnerability index was obtained by adding up the factors related to human well-being that influence individuals exposed to and affected by a hazard, according to data from 2010 Population Census, by IBGE. The values related to the hazard are here called "level of susceptibility to flooding (NS)" and refer to the predominant elevation in each sector of the area. Using the correlation of these indexes obtained by geoprocessing techniques, it was possible to determine and prioritize areas of higher or lower risk of flooding. In general, the sectors inhabited by the most vulnerable groups are also the closest to the river floodplain, therefore, highly susceptible to flooding. Altogether, more than 10 thousand people are at high risk of flooding, and more than half of which are women and approximately 2,500 are children. Poverty is also an important factor for those living in these sectors, making their access to clean water, sanitation, infrastructure and safe shelter more difficult. This methodology, which can be applied to other similar situations, can be used to develop risk management criteria, mainly regarding planning and prioritizing actions. Other variables should also be tested in further studies.

Keywords: Risk management; social vulnerability; flooding; Tietê River Plain; Jardim Pantanal.

\section{INTRODUÇÃO}

Ulrich Beck, sociólogo alemão, em sua obra Risk Society, de 1992, descreve a passagem da sociedade industrial para a sociedade do risco, considerando a tendência de generalização de riscos, perigos, vulnerabilidades, exposições, incertezas, inseguranças e medos, elementos típicos da sociedade atual, devido ao aumento da concentração populacional e do crescimento demográfico em áreas urbanas ambientalmente instáveis e socialmente vulneráveis (BECK 2011). Nesse contexto, a gestão do risco é extremamente importante para a prevenção e controle de desastres naturais ou tecnogênicos - provenientes de processos humanos.

A Política Nacional de Proteção e Defesa Civil (PNPDEC), instituída pela Lei Federal 12.608 , de 10 de abril de 2012, propõe um conjunto de ações de prevenção, mitigação, preparação, resposta e recuperação destinadas à gestão de riscos. Para que esta gestão seja eficaz é preciso, antes de mais nada, conhecer o risco, ou seja, a probabilidade de ocorrerem consequências danosas como resultado das interações entre um perigo e as condições de vulnerabilidade local.

O termo perigo é entendido como um fenômeno potencialmente danoso, o qual pode causar perdas de vidas, ferimentos às pessoas, danos às propriedades, interrupções de atividades sociais e econômicas ou degradação ambiental, originário de processos naturais (geológicos, hidrometeorológicos e biológicos) ou induzido por processos humanos, quantificado em termos de magnitude ou frequência (CASTRO 1999, UN-ISDR 2002, AMARAL \& GUTJAHR 2011).

Por sua vez, a vulnerabilidade refere-se ao grau ou à condição em que as comunidades humanas, devido à exposição ao perigo, encontram-se sujeitas a danos - ou seja, quando há um potencial de perda. Isso depende da capacidade dessas populações responderem e se recuperarem das consequências dos desastres. Ela é determinada por uma combinação de fatores que se concentram principalmente nos aspectos de organização social, coletivos, e incluem a percepção da população acerca dos perigos, as condições de vida nos assentamentos e a infraestrutura existente, bem como a efetividade das políticas e da gestão pública. Portanto, seu entendimento requer uma integração multidisciplinar de conhecimentos, provenientes tanto das geociências quanto das ciências humanas (CUTTER et al. 2003, CUTTER 2011, PELOGGIA 2011, IPCC 2012).

Assim, investigar a correlação entre vulnerabilidade, perigo e risco contribui para a boa gestão, subsidiando a tomada de decisões e a busca por medidas preventivas e mitigadoras para os desastres, diante da eventual impossibilidade de realocação completa dos moradores que vivem em áreas de risco ou da redução do grau de perigo, em atendimento à PNPDEC. Nesse contexto, a vulnerabilidade pode ser entendida como um critério objetivo para a análise multidimensional de riscos (PELOGGIA 2011), uma vez que pode ser mensurada, em áreas definidas, por meio de parâmetros como densidade demográfica, renda, número de habitantes expostos 
à situação de perigo e composição etária da população afetada, entre outros indicadores.

Neste trabalho será abordado o caso do Jardim Pantanal, uma área de risco sujeita a inundações, ocupada principalmente por assentamentos irregulares, altamente adensada e localizada na antiga várzea do rio Tietê, município de São Paulo (SP). Trata-se de um compartimento de relevo cujas expressivas transformações antropogênicas, como acúmulo de depósitos tecnogênicos e alterações da dinâmica fluvial, permitem caracterizá-lo como planície tecnogênica (PELOGGIA 1998, 2005; PELOGGIA et al. 2014a). O local tem sido alvo de interesse constante da mídia por permanecer alagado durante longos períodos, situação frequente que ocorre desde o início da década de 1990. Assim, o objetivo é determinar a variação da vulnerabilidade social da área de estudo frente a tal situação de risco, de forma a se definir uma metodologia de hierarquização de risco aplicável como ferramenta de gestão.

\section{2 ÁREA DE ESTUDO}

Jardim Pantanal é a designação informal, porém consagrada pela população e pela mídia, de um assentamento espontâneo que sofre com frequentes eventos de inundações, localizado no distrito do Jardim Helena, na Subprefeitura de São Miguel Paulista, Zona Leste da cidade de São Paulo, próximo à divisa com os municípios de Guarulhos e Itaquaquecetuba. Não se sabe, ao certo, a delimitação do Jardim Pantanal, e muitos acreditam, influenciados principalmente pela mídia, que o Jardim Pantanal é toda área de várzea do Jardim Helena, ou mesmo confundem com o Jardim Romano, localizado a extremo leste do distrito. O Instituto Alana, uma organização não governamental sem fins lucrativos, localizada no Jardim Pantanal e existente há mais de 10 anos, propõe uma delimitação seguindo os setores censitários do IBGE (Figura 1).

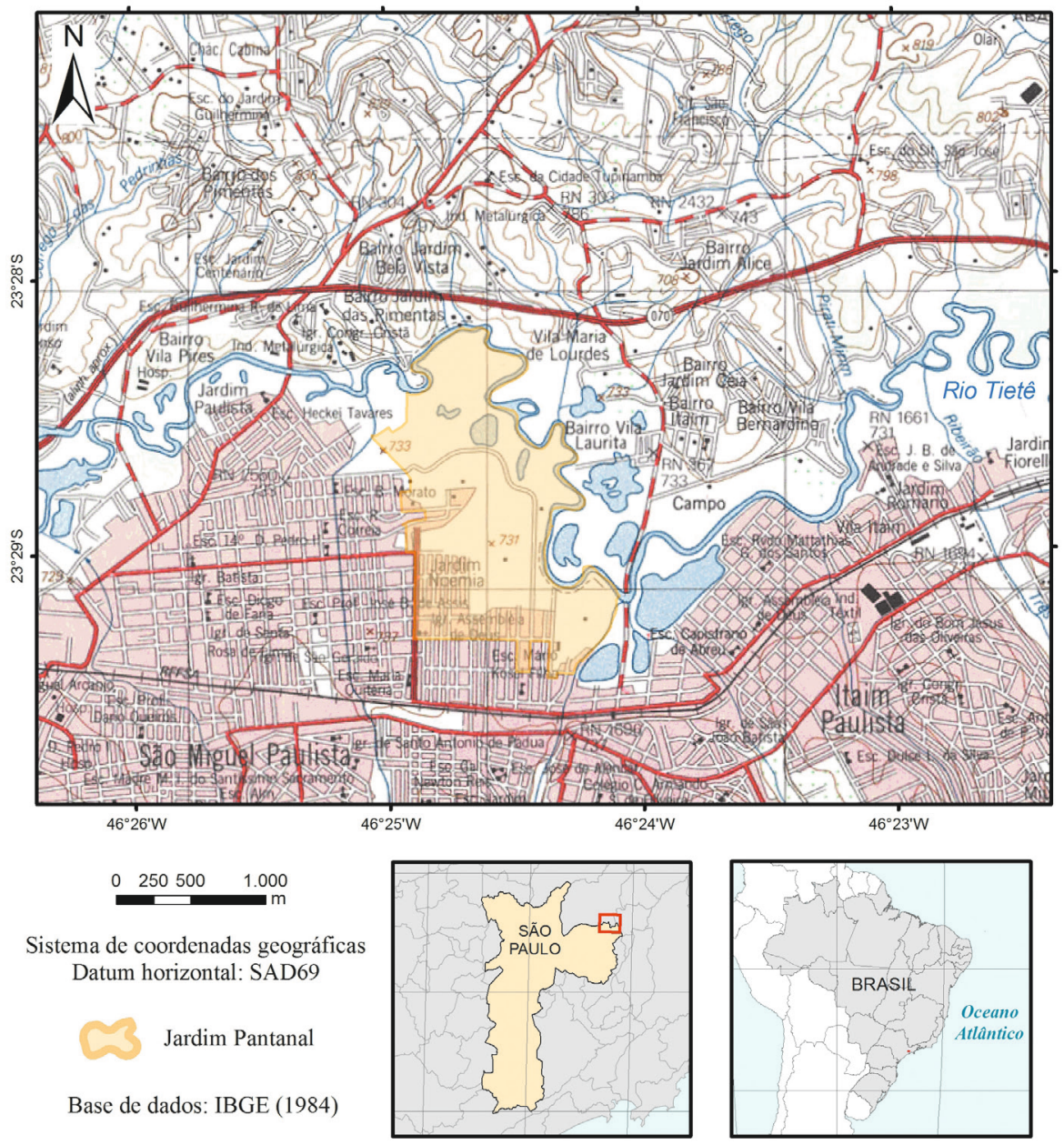

FIGURA 1 - Delimitação do Jardim Pantanal, zona leste da cidade de São Paulo, feita sobre a Carta 1:50.000 (Itaquaquecetuba) do IBGE, de 1981, evidenciando a configuração do local antes da ocupação. 
O local situa-se em um compartimento de relevo de planície aluvial modificada pela ação humana, caracterizada por terrenos baixos e mais ou menos planos, junto às margens de meandros remanescentes do rio Tietê. A configuração geológica original consiste em formações aluvionares recentes, caracterizadas pela presença de turfas, argilas com ou sem matéria orgânica, siltes, areias e cascalhos. Ressalta-se, todavia, a ampla formação de terrenos tecnogênicos de aterramento e preenchimento, realizados ao longo do processo de ocupação do local. A várzea originalmente funcionava, naturalmente, como leito maior do rio, estando, portanto, sujeita a inundações sazonais. (IPT 1981, 1985, 1994; PELOGGIA et al. 2014b)

A ocupação das várzeas do Tietê, no município de São Paulo, foi decorrente do processo de expansão da cidade ao longo do século XX, no bojo do qual se retificaram os principais rios da cidade, o que propiciou a drenagem e aterramento das planícies, criando-se assim um conjunto de terrenos artificiais ou tecnogênicos, que foram progressivamente urbanizados (PELOGGIA 1998, 2005). A região do Jardim Pantanal está localizada no único trecho do rio Tietê no município, onde ainda se observam meandros não retificados, apesar da planície ter sido extensamente aterrada e ocupada por assentamentos de baixo padrão construtivo (Figura 2). Situada a montante da barragem da Penha, abrigando ainda pântanos e brejos, a área funciona como um instrumento hidráulico regulador do escoamento das águas no trecho retificado, evitando enchentes nas vias marginais aos canais do Tietê e do Pinheiros e outros pontos da cidade (SUENAGA \& PUNTONI 2010).

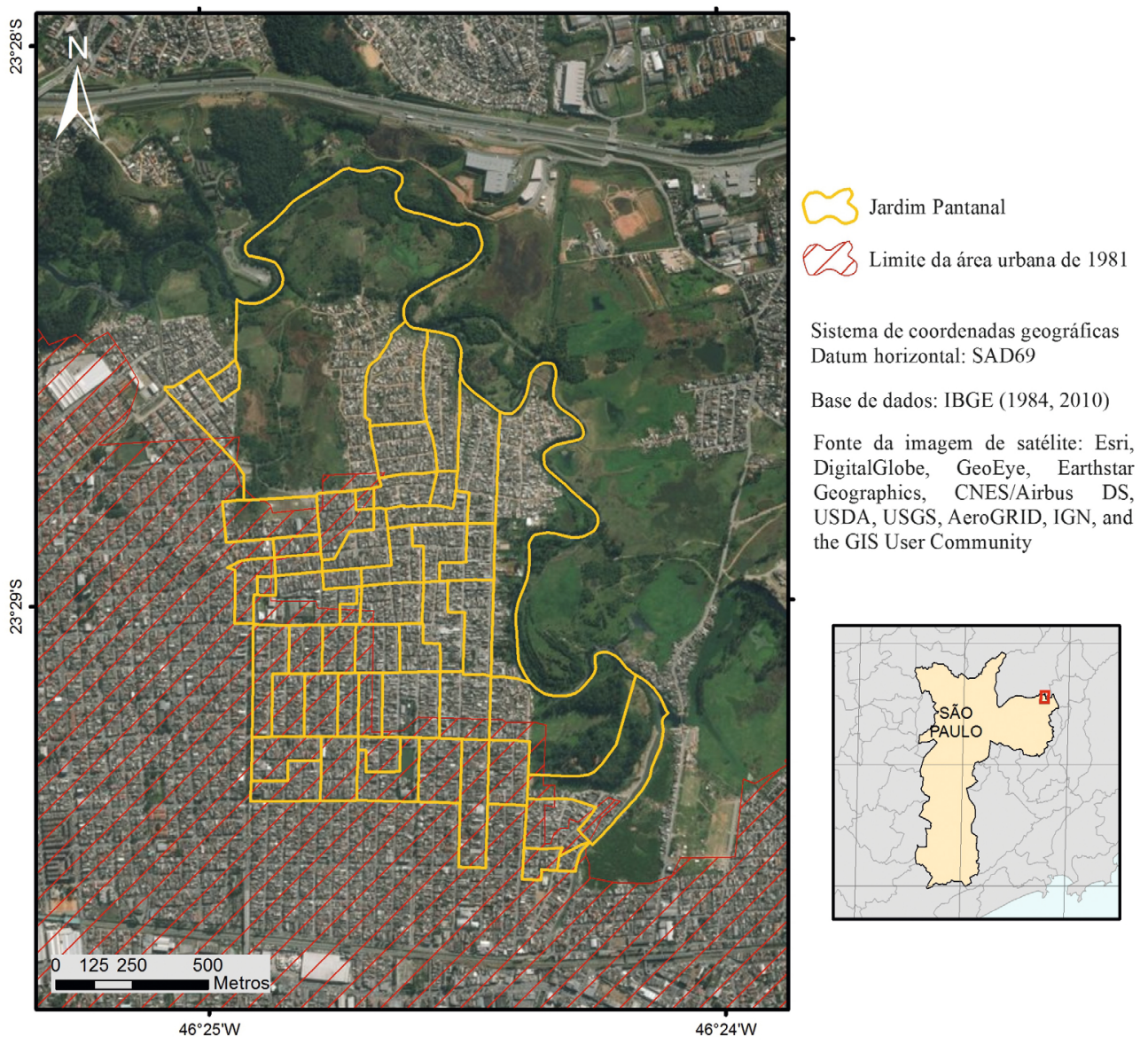

FIGURA 2 - Imagem de satélite com delimitação do Jardim Pantanal, evidenciando o adensamento populacional ocorrido a partir da década de 1980. 
O assentamento estudado surgiu entre meados dos anos 1980 e início da década de 1990 e é resultado de ocupação irregular acarretada pelo déficit habitacional presente na época (CRISTIANO et al. 2012). Na década de 1990, o loteamento cresceu desordenadamente, ocupado por uma comunidade bastante carente (PONCIANO 2004). Nos últimos anos, além de novos loteamentos, algumas obras de infraestrutura foram implantadas na região. Algumas ruas foram pavimentadas, mas em geral, não há galerias de águas pluviais instaladas, intensificando o problema de escoamento das águas pluviais. Além disso, no Jardim Romano, a montante do Jardim Pantanal, foi implantada uma grande intervenção hidráulica para contenção de inundações, com um dique, um pôlder e um reservatório. Segundo informações do Instituto Alana (Acervo institucional: Jardim Pantanal. São Paulo), a obra não teria diminuído a frequência de ocorrência das inundações, apenas acelerado o processo de escoamento da água no Jardim Pantanal.

A primeira grande inundação registrada no local aconteceu em 1992, quando o número de moradores ainda era baixo. A partir de notícias de jornal, foi realizado um levantamento dos eventos de inundação ocorridos na região, tendo sido contabilizados, ao longo de 20 anos, onze casos graves (registrados no acervo da Folha de S. Paulo): 1992, 1995, 1996, 1997, 1999, 2005, 2009, 2010 e 2012. Tais dados podem ser considerados subestimados e provavelmente não correspondem à realidade, uma vez que nem todas as inundações ocorridas podem ter sido noticiadas.

Esses registros indicam diversos danos causados pela inundação, como desabamentos, desabrigados, doenças e vítimas fatais, além de reflexos no trânsito e no transporte público. O noticiário destaca que a região permanece alagada por vários dias consecutivos, o fato de ser uma ocupação irregular, a falta de infraestrutura, a possibilidade de desocupação e a hipótese de que as inundações são provocadas pelo fechamento da barragem da Penha (segundo as matérias, o DAEE teria negado a eventual relação entre as inundações no Jardim Pantanal e a barragem).

Em particular, o caso do Jardim Pantanal ganhou muito destaque durante as fortes chuvas do verão de 2009/10, em que o bairro ficou alagado mais de 60 dias. Moradores tiveram suas casas invadidas pelas águas poluídas do rio Tietê e não teriam tido respaldo do poder público, de acordo com a população local (CRISTIANO et al. 2012). $\mathrm{Na}$ ocasião, aproximadamente 340 famílias foram removidas às pressas para conjuntos habitacio- nais em Itaquaquecetuba, município vizinho de São Paulo. Outras três mil famílias aguardavam as moradias prometidas após dois anos. Ao todo, foram 9.907 pessoas atingidas pela inundação, sendo que dois adolescentes morreram, famílias perderam todos os pertences, o risco de infecções por doenças como leptospirose foi altíssimo, as escolas fecharam, além do que era comum a presença de animais peçonhentos na água, aumentando a insegurança dos moradores (FOLHA DE SÃO PAULO 2012). O Decreto Municipal paulistano $\mathrm{n}^{\circ} 51.225$, de 02 de fevereiro de 2010 declarou a inundação de 2009/10 como estado de calamidade pública, estipulando um prazo para que fossem realizadas ações de mitigação.

\section{FUNDAMENTAÇÃO TEÓRICA E DESENVOLVIMENTO DO MÉTODO DE ANÁLISE}

VILLA \& MCLEOD (2002) apontam três passos necessários à construção de um método de avaliação da vulnerabilidade ambiental: definição do conceito de vulnerabilidade, escolha do sistema a ser avaliado e escolha e organização dos indicadores ambientais. A necessidade de se definir o conceito de vulnerabilidade advém da existência de diferentes concepções sobre o tema, que implicam em diferentes abordagens de análise. O uso científico do termo tem suas raízes na pesquisa da geografia e dos riscos naturais, no entanto, é muitas vezes utilizado com foco em sistemas sociopolíticos, aplicado como um conceito central em risco de desastres, no estudo de meios de subsistência e da pobreza, segurança alimentar, saúde pública e das mudanças climáticas (MILLER \& BOWEN 2013).

De fato, a referência à vulnerabilidade na literatura é feita há várias décadas e diversas definições podem ser encontradas a seu respeito (ver TAYLOR \& BUTTERFIELD 2011, para uma revisão sobre o assunto). Conforme definição adotada pela PNPDEC (Lei 12.608/2012), vulnerabilidade é uma condição intrínseca ao corpo ou sistema receptor que, em interação com a magnitude do evento ou acidente, caracteriza os efeitos adversos, medidos em termos de intensidade dos danos prováveis. É uma relação existente entre a magnitude da ameaça, caso ela se concretize, e a intensidade do dano consequente. Por outro lado, SILVA (2012) descreve vulnerabilidade como o grau de resistência ou suscetibilidade de um sistema socioeconômico em relação ao impacto 
dos perigos naturais e desastres tecnológicos ou ambientais. É, portanto, um fator influenciado pelas ações e comportamento humano.

Dentre as diversas abordagens do termo vulnerabilidade, identifica-se um elemento comum: a ameaça (ou perigo). Por exemplo, as ameaças industriais, tecnológicas, sanitárias, naturais (mudanças climáticas), ecológicas, entre outras. De maneira geral, o termo vulnerabilidade é um aspecto voltado a um sujeito ou sistema (humano ou não). A esses, o uso comum da palavra refere-se à sua capacidade de ser suscetível a um desastre devido à exposição a um perigo.

O IPCC (2012) define as três componentes da vulnerabilidade: exposição, sensibilidade e capacidade de adaptação. $\mathrm{O}$ estudo desses fatores permite a avaliação da maior ou menor vulnerabilidade de um sistema a determinadas questões ambientais. A exposição significa o grau, duração ou extensão em que o sistema está em contato com perturbações. A sensibilidade está relacionada à extensão ou ao grau em que um sistema pode absorver as pressões sem sofrer alterações no longo prazo. A capacidade adaptativa é a habilidade do sistema de se ajustar a um dano ocorrido, de fazer uso de recursos ou oportunidades ou responder a mudanças ambientais que venham a ocorrer. Nesse contexto, um sistema é mais vulnerável quanto maior a exposição, maior a sensibilidade do meio e menor sua capacidade adaptativa (ADGER 2006).

$\mathrm{O}$ estudo da vulnerabilidade também requer a delimitação do sistema ambiental em estudo, que fornecerá indicadores relativos aos processos atuantes e à suscetibilidade. O Jardim Pantanal é parte de um sistema tecnogênico específico, cujos agentes atuam de forma integrada na composição da paisagem e na alteração da mesma. Nesse contexto, diversos estudos têm detectado um aumento na intensidade e na frequência de dias com chuvas intensas e concentradas em curto período de tempo na Região Metropolitana de São Paulo, eventos que podem gerar inundações e deslizamentos de terra em áreas de risco (MCTI 2013). Além do aumento das chuvas, FARIA (2010) destaca que as alterações no meio são condicionantes tecnogênicas de inundações, destacando o uso e ocupação irregular nas planícies e margens de cursos d'água, a disposição irregular de resíduos, as alterações nas características da bacia hidrográfica e o intenso processo de erosão das encostas e consequente assoreamento dos canais. Essa situação é observada na área de estudo, consistindo-se em um grave problema para a população local.
O método de análise proposto neste trabalho, detalhado na próxima seção, é baseado na proposta inicial apresentada por MALAGODI \& PELOGGIA (2013), que considera como indicadores fatores relacionados ao bem-estar humano que integra os indivíduos expostos e afetados por um perigo de uma área e a sensibilidade do sistema. A vulnerabilidade distingue-se entre grupos sociais por apresentarem diferentes graus de exposição ou vulnerabilidade a eventos extremos, podendo apresentar capacidade de adaptação mais limitada e serem mais dependentes de recursos sensíveis ao clima, como a oferta local de água e alimento. São grupos sociais mais frágeis, como por exemplo: idosos, crianças, mulheres, indivíduos ou grupos de baixa renda e baixa escolaridade, e sistemas que apresentem deficiência na gestão e implementação de políticas públicas, falta de investimentos na saúde e melhoria no saneamento, segurança alimentar, manutenção da gestão de resíduos sólidos e infraestrutura.

Tal abordagem apresenta uma configuração e estrutura lógica muito semelhante à desenvolvida por GOERL et al. (2012), que foi aplicada ao mapeamento de áreas de risco no município de Negrinho (SC), sendo, contudo, os indicadores abordados de forma diferenciada. A escolha de indicadores para uma análise de vulnerabilidade requer levar em consideração critérios como mensurabilidade, relevância, caráter analítico, disponibilidade de dados, comparabilidade, capacidade de ser reproduzida em outras pesquisas, entre outros.

Deve-se considerar inicialmente que a análise de riscos ambientais (na qual se inserem as situações de risco geológico e risco de inundação) pode ser tratada por meio de análises e abordagens diversas, em escalas territoriais diferenciadas, seja em nível estadual (e.g. MARCELINO et al. 2006), municipal (e.g. GOERL et al. 2012) ou local (e.g. MALAGODI \& PELOGGIA 2013, e onde se insere este trabalho). Cada um desses níveis de análise exige a mobilização de indicadores diferenciados, compatíveis com a escala e os objetivos. No entanto, tomando-se os elementos essenciais do problema, duas variáveis devem ser consideradas: a primeira indica a possibilidade ou a probabilidade de ocorrência de um fenômeno potencialmente destrutivo (referindo-se, portanto, à caracterização de uma ameaça geradora de perigo) e se refere à suscetibilidade espacialmente diferenciada de deflagração do mesmo. Já a segunda variável se refere às consequências potenciais, o que implica em primeiro lugar à exposição ao perigo, mas também depende da vulnerabilidade e da capacidade de resposta e 
recuperação, ou resiliência, das comunidades ou sistemas sociais afetados.

Considerando os conceitos propostos por BITAR et al. (2014), o risco é determinado pela conjugação de elementos relativos a processos ambientais que criam zonas de suscetibilidade e de processos sociais que implicam no surgimento de zonas de ocupação sobrepostas às de suscetibilidade, surgindo assim, zonas de elementos expostos, ou seja, as áreas de risco. É a natureza e a intensidade da ameaça, associada à abrangência da exposição, à fragilidade ou capacidade de absorção de impacto, resposta e recuperação de um sistema social, que determinarão a intensidade do risco. Desta forma, a análise de risco pode ser expressa pela equação 1, proposta pelo UN-ISDR (2002), a seguir.

$$
\mathrm{R}=\frac{P V}{R e}
$$

(Equação 1)

Onde: $R$ é o risco, $P$ é o perigo, $V$ é a vulnerabilidade e $R e$ é a resposta.

MARCELINO et al. (2006) lembram que, no caso de desastres associados às instabilidades atmosféricas, apesar dos danos e prejuízos gerados, estes não ocasionam um número tão elevado de vítimas fatais, mas sim de desabrigados. Assim, visando fornecer um indicativo do grau de risco de pessoas afetadas por localidade, foi proposta pelos autores supracitados, uma adaptação da equação 1 :

$$
\mathrm{R}=\frac{P(D D+P I+I P)}{I D H M}
$$

Onde: $R$ é o risco, $P$ é o perigo, $D D$ é a densidade demográfica, $P I$ é a população idosa, $I P$ é a intensidade da pobreza e $I D H M$ é o índice de desenvolvimento humano municipal. $\mathrm{O}$ fator $P$ é expresso pelo número de eventos ocorridos por ano, $D D$ é a razão entre a população residente total e a área do município $\left(\right.$ hab $\left./ \mathrm{km}^{2}\right), I P$ é expresso pela renda per capita dos setores censitários, $P I$ é o número de pessoas com 65 anos ou mais e $I D H M$ é obtido pela média aritmética de três sub-índices referentes às dimensões longevidade (IDH-Longevidade), educação (IDH-Educação) e renda (IDH-Renda).

Diversas outras metodologias são encontradas na literatura, sendo que o ponto comum entre elas é a opinião de que as atividades de gestão de riscos exigem profissionais com visão interdisciplinar abrangente e multilateral, capazes de dialogar com técnicos e gestores de diversas especialidades, com a população e com os administradores públicos e autoridades, compreendendo seus pontos de vista e, ao mesmo tempo, influenciando-os e orientando-os no sentido das melhores soluções de gestão (PELOGGIA 2011).

O termo exposição é empregado, segundo o IPCC (2012), para se referir à presença (localização) de pessoas, meios de vida, serviços e recursos ambientais, infraestrutura, ou bens econômicos, sociais ou culturais em locais que possam ser adversamente afetados por eventos físicos e que, portanto, estão suscetíveis a potenciais futuros desastres. No entanto, na literatura é comum se confundir os termos exposição e vulnerabilidade. A exposição é um fator determinante necessário, mas não suficiente, de risco. É possível ser exposto, mas não ser vulnerável.

Conforme IPCC (2012), vulnerabilidade e exposição são aspectos dinâmicos e dependem de fatores econômicos, sociais, demográficos, culturais, institucionais e governamentais. Indivíduos e comunidades são diferencialmente expostos com base em fatores como riqueza, educação, sexo, idade, classe/casta e saúde. Por exemplo, as pessoas que têm percepção, meios de vida transferíveis, dinheiro e acesso a transporte, podem afastar-se dos desastres e viver mais confortavelmente, distantes do perigo. As que não possuem essas vantagens podem ser forçadas a fixar suas residências em áreas passíveis de risco, onde ficarão mais vulneráveis e expostas aos extremos climáticos.

\section{MÉTODO DE ANÁLISE DA VULNERABILIDADE APLICADO}

Para definir a vulnerabilidade da área de estudo, adotou-se o método proposto pela UN-ISDR (2002), tendo como referência o estudo de caso de MARCELINO et al. (2006). Assim, a partir das equações 1 e 2 , e com base na fundamentação teórica desenvolvida por MALAGODI \& PELOGGIA (2013), foi proposta a seguinte equação para o cálculo da vulnerabilidade social a ser aplicado em cada setor censitário do Jardim Pantanal.

$$
V S=D D+P M+P C+P I+I P \quad \text { (Equação 3) }
$$

Onde: $V s$ é a vulnerabilidade social, $D D$ é a densidade demográfica, $P M$ é a população de mulheres, $P C$ é a população de crianças, $P I$ é a população idosa e $I P$ é a intensidade da pobreza. Tais indicadores sociais foram determinados a partir de dados do censo demográfico de 2010 disponibilizados pelo IBGE, por setor censitário. 
Após os cálculos, foi estabelecido um índice para determinar o grau de vulnerabilidade dos setores, reclassificado entre 0 e 1 (máxima vulnerabilidade), a partir da seguinte equação:

$$
\text { Índice }=\frac{(\text { observado }-V \text { mínimo })}{(\text { V máximo }- \text { V mínimo })} \quad(\text { Equação } 4)
$$

No entanto, em função dos resultados obtidos no estudo desenvolvido por MALAGODI \& PELOGGIA (2013), verificou-se a necessidade de uma adequação metodológica para a determinação da vulnerabilidade social, calculada a partir da soma dos fatores sociodemográficos absolutos. Após uma série de testes, observou-se que o cálculo da vulnerabilidade deveria considerar os valores relativos dos indicadores ao invés dos absolutos, pois desse modo o peso dos fatores seria ponderado, permitindo comparações mais acuradas. Da mesma forma, o fator $I P$, relacionado à renda, foi aprimorado utilizando-se os dados da renda per capita por setor censitário disponibilizados pelo IBGE (2010), o qual, no estudo referido, foi utilizado um valor neutro para todos os setores censitários (1). Esse índice varia de 1 (valor mínimo) a 0 (valor máximo), uma vez que quanto menor a renda do setor, maior será a sua vulnerabilidade.

Para exemplificar esta adequação, a tabela 1 apresenta os dados do setor censitário 50 , e os valores de vulnerabilidade social calculados a partir de valores absolutos e relativos. Os índices de vulnerabilidade social foram obtidos a partir da reclassificação dos valores para a escala entre 0 e 1 .

Considerando os valores absolutos, a $V S_{50}$ obteve como resultado o valor de 0,21 (baixa vulnerabilidade social). Por outro lado, adotando-se os valores relativos, o resultado passa a ser 0,49 , caracterizando o setor como mais vulnerável perante o risco a inundação.
Para a determinação do risco, considerou-se o indicador NS (nível de suscetibilidade dos setores à inundação) como representante do fator $P$ (perigo) da equação 1 . O risco $(R)$ dos setores censitários foi determinado pela equação 5 , mostrada a seguir.

$$
R=P V=N S(D D+P M+P C+P I+I P)
$$

(Equação 5)

Conforme a fundamentação teórica, o perigo é mensurado por magnitude ou frequência de eventos ocorridos; no entanto, a série histórica de ocorrências levantada pela Defesa Civil da cidade de São Paulo não é suficiente para representar a exposição ao risco da área de estudo. Da mesma forma, a carta de suscetibilidade à inundação do município de São Paulo (IPT/CPRM 2014) apresenta uma resolução inadequada para ser utilizada em uma escala local. Assim, os valores assumidos por $N S$ referem-se à cota topográfica predominante em cada setor censitário, extraída do Modelo Digital de Elevação (MDE) de resolução de 3 arco-segundo, atualizado em novembro de 2011 (Topodata, INPE 2011). O modelo foi trabalhado no software de Sistema de Informações Geográficas ArcGIS 10.2, por meio da ferramenta 3D Analyst Tools, determinando-se assim as curvas de níveis da área de estudo com o espaçamento de $1 \mathrm{~m}$. A partir da determinação da cota altimétrica predominante em cada setor censitário, gerou-se o índice de suscetibilidade ao risco (exposição) para cada setor (Figura 3), sendo atribuído valor máximo (5) à menor cota (maior exposição ao perigo - suscetibilidade Muito Alta) e mínimo (1) à maior (menor exposição - suscetibilidade Muito Baixa).

Da mesma forma que para a vulnerabilidade social, a variável $N S$ foi reclassificada entre 0 e 1 , a partir da equação 4 . A tabela 2 exemplifica a determinação do risco para o setor 50 .

TABELA 1 - Exemplo de adequação metodológica relativa ao cálculo de vulnerabilidade social para o setor

\begin{tabular}{|c|c|c|c|c|c|c|c|}
\hline $\begin{array}{c}\text { Setor } \\
\text { Censitário } \\
\text { ID } 50\end{array}$ & $\begin{array}{l}\text { Densidade } \\
\text { Demográfica } \\
(\text { hab/m².10 }\end{array}$ & $\begin{array}{l}\text { Mulheres } \\
\text { (hab) }\end{array}$ & $\begin{array}{c}\text { Crianças } \\
\text { (hab) }\end{array}$ & $\begin{array}{c}\text { Idosos } \\
(h a b)\end{array}$ & $\begin{array}{c}\text { Renda média } \\
\text { per capita } \\
(R \$)\end{array}$ & $\begin{array}{c}\text { Vulnerabilidade } \\
\text { Social }\end{array}$ & $\begin{array}{c}\text { Índice de } \\
\text { Vulnerabilidade } \\
\text { Social }\end{array}$ \\
\hline $\begin{array}{l}\text { Valores } \\
\text { absolutos }\end{array}$ & 53,16 & 207 & 117 & 7 & 664,13 & $1.048,29$ & 0,21 \\
\hline $\begin{array}{l}\text { Valores } \\
\text { relativos }\end{array}$ & 1,0 & 0,19 & 0,24 & 0,01 & 0,80 & 2.24 & 0,49 \\
\hline
\end{tabular}
censitário 50.

$V S_{50}=D D+P M+P C+P I+I P$

$V S_{50}=53,16+207+117+7+664,13=1.048,29$ 

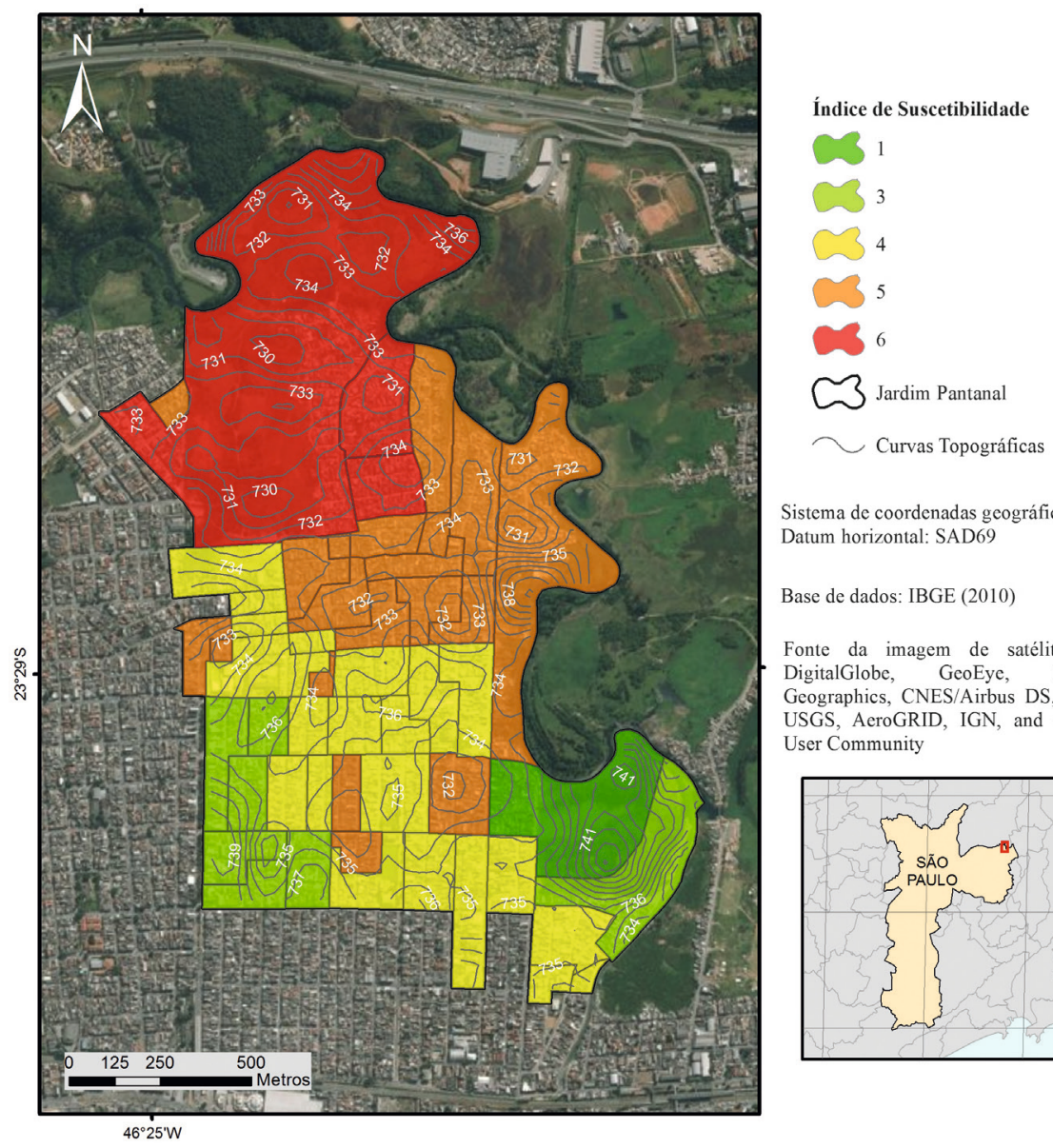

Sistema de coordenadas geográficas Datum horizontal: SAD69

Base de dados: IBGE (2010)

Fonte da imagem de satélite: Esri, DigitalGlobe, GeoEye, Earthstar Geographics, CNES/Airbus DS, USDA, USGS, AeroGRID, IGN, and the GIS User Community

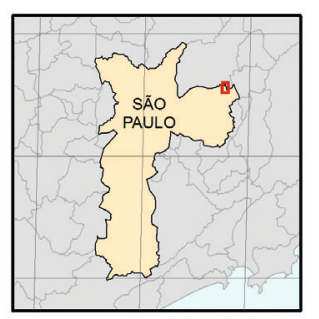

FIGURA 3 - Mapa de Suscetibilidade à Inundação no Jardim Pantanal.

TABELA 2 - Exemplo de cálculo para determinação do risco $(R)$ para o setor censitário 50.

\begin{tabular}{cccccc}
\hline $\begin{array}{c}\text { Setor Censitário } \\
\text { ID 50 }\end{array}$ & $\begin{array}{c}\text { Altitude } \\
\text { predominante }(m)\end{array}$ & $\begin{array}{c}\text { Nivel de } \\
\text { Suscetibilidade }\end{array}$ & $\begin{array}{c}\text { Índice do Nivel de } \\
\text { Suscetibilidade }\end{array}$ & $\begin{array}{c}\text { Índice de } \\
\text { Vulnerabilidade } \\
\text { Social }\end{array}$ & Risco \\
\hline Valores & 733 & 5 & 1,0 & 0,49 & 0,49 \\
\hline
\end{tabular}

Assim, para o setor censitário 50, observa-se que tanto a vulnerabilidade social como o risco de ocorrência de um desastre são altos, uma vez que apresenta elevada densidade demográfica, elevado grau de pobreza e se encontra a uma altitude favorável à ocorrência de novas inundações em comparação a outros setores.

Dentre as variáveis utilizadas para a análise de risco, o fator $R e$ (capacidade de resposta ou resiliência de um sistema) não foi abordado neste trabalho e será foco de estudos futuros. Nesse âmbito, deve-se considerar a análise da percepção do sistema suscetível à situação de risco, que influencia de maneira direta a capacidade de resposta de uma dada comunidade. A percepção é a maneira como diferentes pessoas encaram e se comportam diante de situações de risco.

Para a análise dos resultados foram gerados mapas de vulnerabilidade social e de risco no software ArcGis 10.2. Cada mapa possui 4 classes: Baixa, Média, Alta e Muito Alta, que foram definidas pelo método do Desvio Quartílico. Este método divide a série de dados em quatro grupos com igual número de ocorrências, cada um compreendendo $25 \%$ do total de valores. Desta forma, o fatiamento é definido quantitativamente, excluindo a subjeti- 
vidade do processo de definição do limiar de corte (RAMOS \& SANCHEZ 2000). Para o índice de vulnerabilidade, os valores correspondentes à Baixa vulnerabilidade estão entre 0 e 0,22 , Média entre 0,23 e 0,33, Alta entre 0,34 e 0,47 e Muito Alta entre 0,48 e 1 . Para o risco, os setores foram classificados em: Baixo risco - índices entre $0 \mathrm{e}$ 0,11 ; Médio risco - entre 0,12 e 0,18 ; Alto risco - entre 0.19 e 0,29 e Muito Alto - entre 0,30 e 1.

\section{RESULTADOS E DISCUSSÃO}

A partir dos dados censitários do IBGE e do Modelo Digital de Elevação foram determinados os fatores para o cálculo analítico da vulnerabilidade social e do risco de inundação para o Jardim Pantanal. Verificou-se que, apesar da área como um todo ser submetida ao mesmo tipo de perigo, apresentando características do meio físico homogêneas e situação social pouco variável (vulnerabilidade social homogênea), a aplicação do método permitiu constatar que a área é de fato diferenciada e que existem setores prioritários para a aplicação de medidas contra desastres ambientais.

Conforme observado na figura 4 , os setores mais vulneráveis e propensos a terem um grande número de afetados na ocorrência de um desastre localizam-se a norte e a leste do assentamento. Apesar dos setores mais adensados do Jardim Pantanal se concentrarem na porção central da área, os setores que apresentam os grupos mais vulneráveis são os localizados mais próximos ao leito do rio, que correspondem às áreas mais suscetíveis à ocorrência de inundações, consequentemente assim, às áreas de risco mais elevado (Figura 5). Ao todo mais de 10 mil pessoas residem nos setores mais vulneráveis, sendo que mais da metade são mulheres e aproximadamente 2.500 são crianças. A pobreza nesses setores também é um fator de destaque, o que intensifica a dificuldade ao acesso à água potável e saneamento, infraestrutura e abrigo seguro, como ilustrado na figura 6 , registrada na época de estiagem (agosto 2013) nas proximidades do setor 45 .

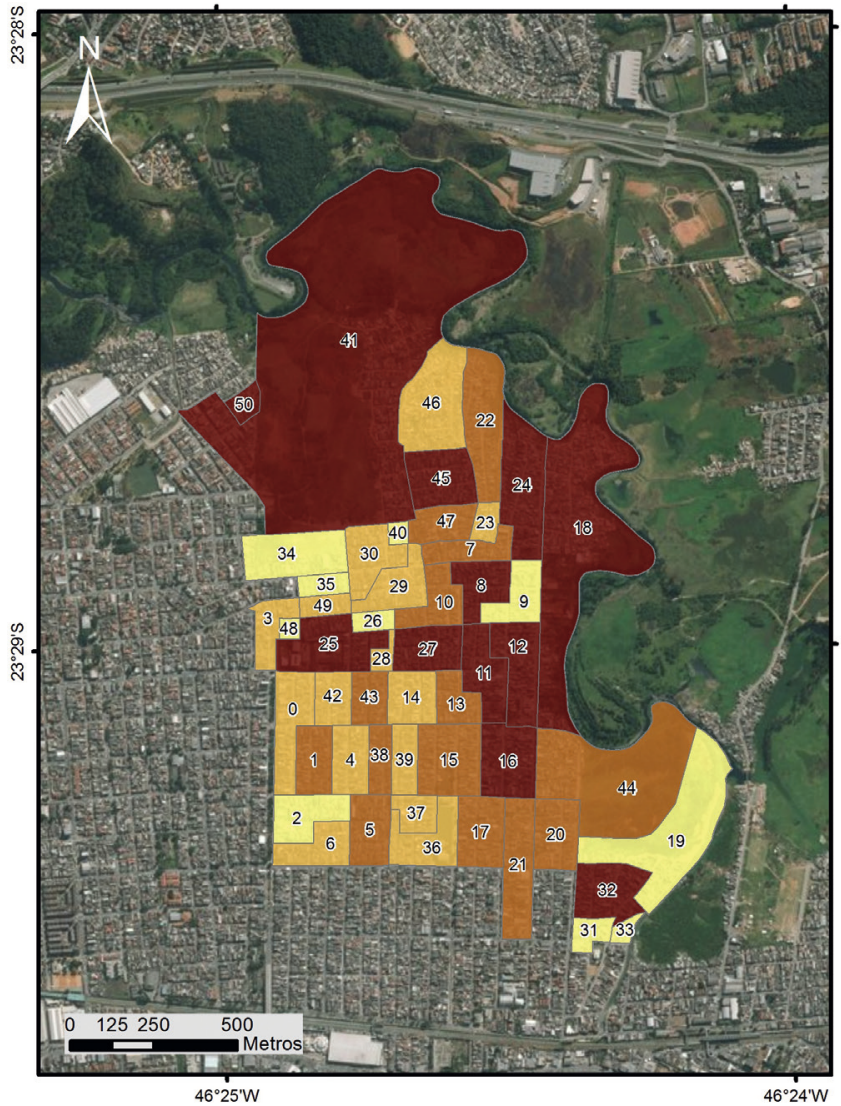

FIGURA 4 - Mapa de Vulnerabilidade Social no Jardim Pantanal.

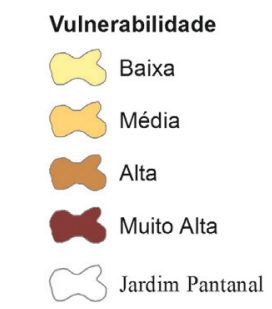

Sistema de coordenadas geográficas Datum horizontal: SAD69

Base de dados: IBGE (2010)

Fonte da imagem de satélite: Esri, DigitalGlobe, GeoEye, Earthstar Geographics, CNES/Airbus DS, USDA, USGS, AeroGRID, IGN, and the GIS User Community

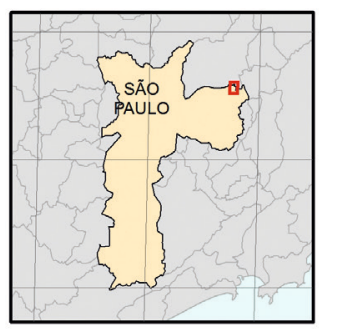



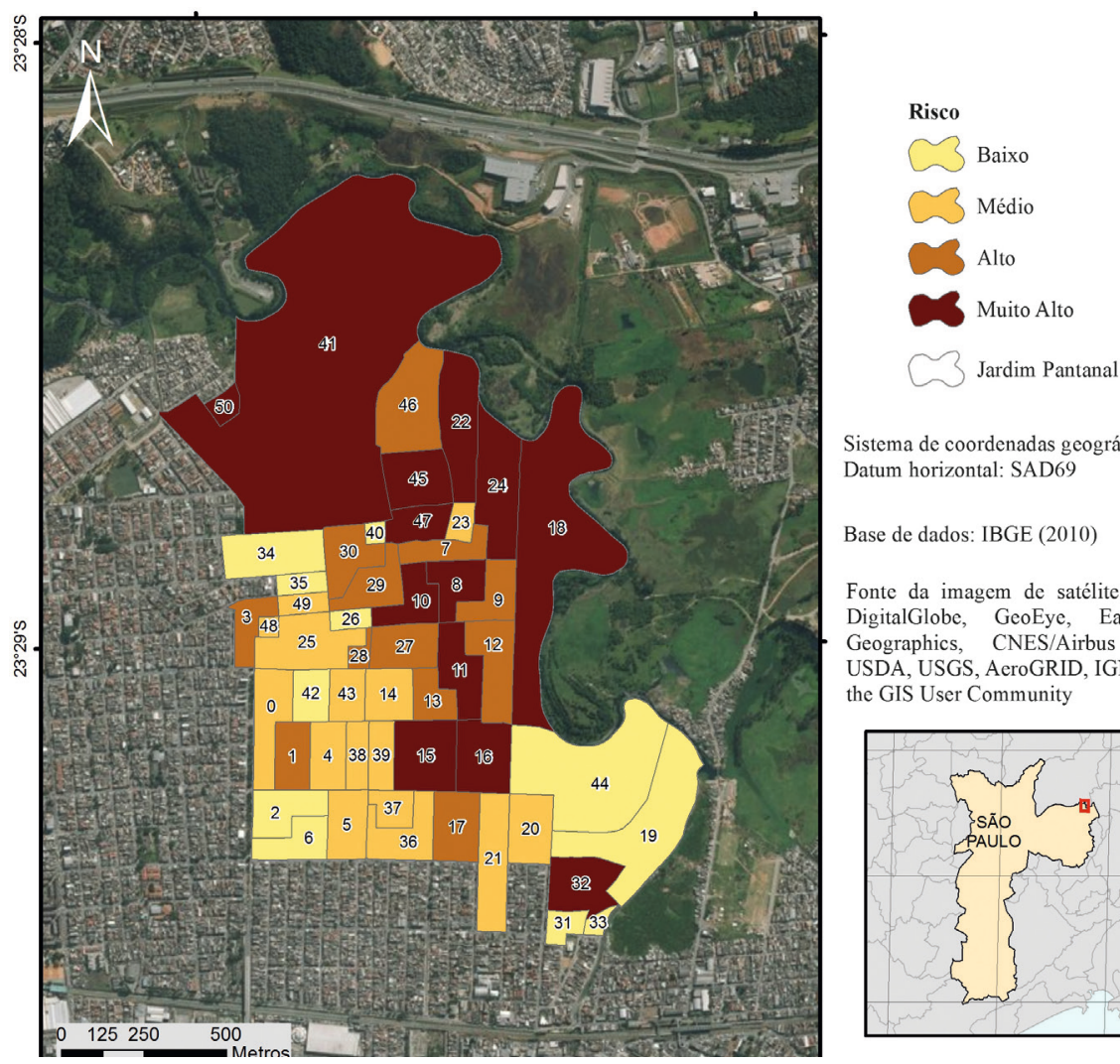

Sistema de coordenadas geográficas Datum horizontal: SAD69

Base de dados: IBGE (2010)

Fonte da imagem de satélite: Esri, DigitalGlobe, GeoEye, Earthstar Geographics, CNES/Airbus DS, USDA, USGS, AeroGRID, IGN, and the GIS User Community

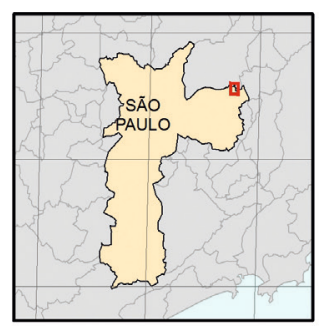

FIGURA 5 - Mapa de Risco de Inundação no Jardim Pantanal.

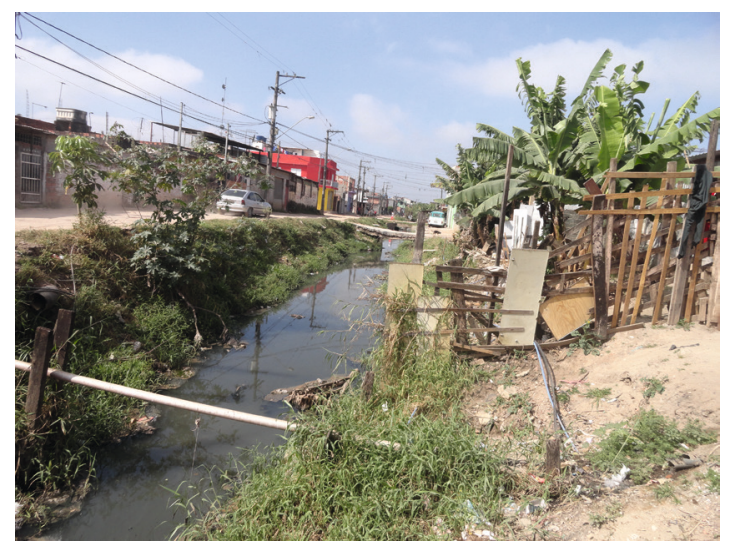

FIGURA 6 - Aspecto da ocupação, evidenciando-se os padrões construtivos e as deficiências de infraestrutura urbana. (Arquivo pessoal de C.C. MALAGODI)

A exposição é um fator determinante e necessário, mas não suficiente, de risco. É possível estar exposto ao desastre, mas não necessariamente vulnerável a ele. Por exemplo, o setor censitário 9 apresenta elevado nível de suscetibilidade, ou seja, está mais exposto a ocorrência de inundações devido a sua cota topográfica, no entanto, não apre- senta dados censitários relevantes para a determinação elevada da vulnerabilidade, devido ao baixo número de pessoas pertencentes aos grupos mais vulneráveis a desastres. Por outro lado, o setor 25 , apesar de apresentar vulnerabilidade social muito alta, está pouco exposto ao perigo (baixo risco de inundação), mas ainda assim é prioritário para o planejamento urbano local.

Em atendimento ao PNPDEC e, principalmente, ao Decreto Municipal $n^{\circ} 51.225$, que estipulam prazos para realização de medidas de intervenção nas áreas de risco, destaca-se a necessidade urgente de medidas para os setores de alto risco do Jardim Pantanal. Na área existem projetos de intervenção urbana em andamento, como o PVT (Programa Parque Várzea do Tietê), o Renova São Paulo e o Plano de Manejo da APA Várzea do Tietê, os quais vêm ao encontro das exigências legais a fim de garantir o acesso dos cidadãos às condições adequadas de moradia, saneamento e infraestrutura. Os setores de maior intervenção coincidem com os aqui identificados como de maior vulnerabilidade e risco. Os projetos respeitam as exigências de se manter a Área de 
Proteção Permanente, recuperando assim, a capacidade de contenção de cheias.

Por fim, deve ser ressaltado que o método desenvolvido é eficiente, mas pode ser refinado. No que diz respeito a variável $R e$ (capacidade de resposta ou resiliência de um sistema), estudos futuros deverão ser realizados para sua inserção na análise de risco, junto com outras variáveis.

\section{CONCLUSÃO}

No presente trabalho aplicou-se um método para determinação dos índices de vulnerabilidade social, perigo e risco associados à ocorrência de inundações no Jardim Pantanal, município de São Paulo, utilizando dados do censo demográfico de 2010 disponibilizados pelo IBGE e informações topográficas derivadas de um modelo digital de elevação.

WATTS \& BOHLE (1993) afirmam que a resposta para a vulnerabilidade é reduzir a exposição, aumentar a capacidade de enfrentamento e reforçar o potencial de recuperação e o controle de danos. Altos níveis de vulnerabilidade e exposição são geralmente o resultado de processos de desenvolvimento assimétrico, associados a gerenciamento ambiental deficiente, mudanças demográficas, urbanização rápida e não planejada, governo falho e escassez de opções de sustento. Isso pode resultar em povoamentos em áreas passíveis de risco, criação de moradias inseguras, favelas e distritos espalhados, pobreza e falta de percepção de riscos. A falta de resistência e capacidade para enfrentar e adaptar-se a extremos são importantes fatores de vulnerabilidade. O Jardim Pantanal é um exemplo de área de risco ocupada de maneira ilegal pela comunidade na busca por moradias, altamente adensada por uma população vulnerável que sofre com frequentes eventos de inundações.

A hierarquização da vulnerabilidade e do risco no Jardim Pantanal permitiu constatar a existência de setores prioritários para a aplicação de medidas contra desastres ambientais. De modo geral, os setores que apresentam os grupos mais frágeis estão alocados em áreas altamente suscetíveis à ocorrência de inundações.

A intensidade dos danos é diretamente proporcional à vulnerabilidade das comunidades afetadas. Desse modo, identificar o grau de vulnerabilidade das comunidades é uma importante medida para se minimizar os prejuízos materiais e humanos decorrentes dos desastres naturais. A determinação do nível de suscetibilidade e do risco do sistema social também são elementos-chaves para a definição de medidas preventivas para uma comunidade enfrentar, resistir e recuperar-se de um desastre natural.

O método proposto no presente estudo, utilizando informações disponíveis em bancos de dados governamentais e recursos geotecnológicos, configura-se em uma ferramenta de baixo custo e aplicação imediata. Sua análise, no entanto, pode ser ampliada com a inserção de dados referentes à percepção de riscos, além de um refinamento no tratamento das variáveis.

\section{AGRADECIMENTOS}

Os autores agradecem os comentários efetuados durante a apresentação deste estudo no $14^{\circ}$ Congresso Brasileiro de Geologia de Engenharia e Ambiental, realizado pela ABGE, e às sugestões e críticas feitas pelos relatores da Revista do Instituto Geológico. Agradecem também ao IBGE, pelo acesso aos dados censitários, ao Instituto Alana, pelo apoio no que tange ao conhecimento da área de estudo e a Defesa Civil do município de São Paulo pelos esclarecimentos.

\section{REFERÊNCIAS BIBLIOGRÁFICAS}

ADGER, W.N. 2006. Vulnerability. Global Environmental Change,16(3): 268-281.

AMARAL, R.; GUTJAHR, M.R. 2011. Desastres naturais (Série Cadernos de Educação Ambiental, 8). IG/SMA, São Paulo, 100 p.

BECK, U. 2011. Sociedade de risco: rumo a uma outra modernidade. Editora 34, São Paulo, $368 \mathrm{p}$.

BITAR, O.A. (coord.) 2014. Cartas de suscetibilidade a movimentos gravitacionais de massa e inundações, escala 1:25.000. Nota técnica explicativa. São Paulo: IPT, DF: CPRM - Serviço Geológico do Brasil.

CASTRO, A.L.C. 1999. Manual de Planejamento em Defesa Civil - Volume I. Ministério da Integração Nacional/Secretaria de Defesa Civil, Brasília, 133 p. Disponível em http://www.defesacivil.mg.gov.br/images/ documentos/Defesa\%20Civil/manuais/ Manual-PLANEJAMENTO-1.pdf. Acessado em 22 mar. 2013. 
CRISTIANO, B.A.; SILVA, V.S.; CARRARO, R. 2012. Repórter multimídia: a grande reportagem na internet. In: INTERCOM, CONGRESSO DE CIÊNCIAS DA COMUNICAÇÃO NA REGIÃO SUDESTE, 17, Ouro Preto, Anais eletrônicos. Disponível em http://www.intercom.org.br/papers/ regionais/sudeste2012/expocom/EX330264-1.pdf. Acessado em 29 nov. 2012.

CUTTER, S.L. 2011. A ciência da vulnerabilidade: modelos, métodos e indicadores. Revista Crítica de Ciências Sociais, 93: 59-69.

CUTTER, S.L;. BORUFF J.B.; SHIRLEY W. L. 2003. Social Vulnerability to Environmental Hazards. Social Science Quarterly, 84(2): 242-261.

FARIA, D.G.M. 2010. Atuação do IG na prevenção de desastres naturais. São Paulo: Instituto Geológico. Ciclo de Palestras em Administração de Emergências para Municípios (CAEM). Disponível em http:// www.igeologico.sp.gov.br/downloads/ palestras/CAEM\%20Registro\%20abril2010. pdf. Acessado em 22 mai. 2013.

FOLHA DE SÃO PAULO. 2012. Após 3 anos, Jardim Romano relembra enchente que durou meses. Folha de S. Paulo, São Paulo, 6 dez. 2012. Disponível em http://mural.blogfolha. uol.com.br/2012/12/06/apos-3-anos-jardimromano-relembra-enchente-que-duroumeses. Acessado em 22 mar. 2013.

GOERL，R.F.; KOBIYAMA， M.; PELLERIN, J.R.G.M. 2012. Proposta metodológica para mapeamento de áreas de risco a inundação: estudo de caso do Município de Rio Negrinho - SC. Boletim de Geografia, 30(1): 81-100.

IBGE - INSTITUTO BRASILEIRO DE GEOGRAFIA E ESTATÍSTICA. 2010. Mapeamento topográfico. Malha do setor censitário. Disponível em http://mapas. ibge.gov.br/bases-e-referenciais/basescartograficas/malhas-digitais. Acessado em 05 mai. 2013.

INPE - INSTITUTO NACIONAL DE PESQUISAS ESPACIAIS. 2011. Topodata: banco de dados geomosfométricos do Brasil. Disponível em http://www.dsr.inpe.br/topodata/index.php. Acessado em 05 mai. 2013.
IPCC - INTERGOVERNMENTAL PANEL ON CLIMATE CHANGE. 2012. Summary for policymakers in: managing the risks of extreme events and disasters to advance climate change adaptation. Cambridge University Press, New York, p. 1-19.

IPT $\quad-\quad$ INSTITUTO DE PESQUISAS TECNOLÓGICAS DO ESTADO DE SÃO PAULO. 1981. Mapa geomorfológico do Estado de São Paulo, escala 1:1.000.000. São Paulo.

IPT - INSTITUTO DE PESQUISAS TECNOLÓGICAS DO ESTADO DE SÃO PAULO. 1985. Carta geotécnica do Município de São Paulo, escala 1:25.000. São Paulo.

IPT - INSTITUTO DE PESQUISAS TECNOLÓGICAS DO ESTADO DE SÃO PAULO. 1994. Carta geotécnica do Estado de São Paulo, escala 1:500.000. São Paulo.

IPT - INSTITUTO DE PESQUISAS TECNOLÓGICAS DO ESTADO DE SÃO PAULO/CPRM - COMPANHIA DE PESQUISA DE RECURSOS MINERAIS. 2014. Carta de suscetibilidade a movimentos gravitacionais de massa e inundações Município de São Paulo, escala 1:75.000. IPT/ CPRM, São Paulo. Revisado em março/2015.

MALAGODI, C.C.; PELOGGIA, A.U.G. 2013. Análise de vulnerabilidade aplicada à gestão de riscos hídricos: estudo de um assentamento urbano na planície de inundação do rio Tietê no município de São Paulo (SP). In: ABGE, CONGRESSO BRASILEIRO DE GEOLOGIA DE ENGENHARIA E AMBIENTAL, 14, Rio de Janeiro, Anais, 10 p. (CD-ROM).

MARCELINO, E.V.; NUNES, L.H.; KOBIYAMA, M. 2006. Mapeamento de risco de desastres naturais do Estado de Santa Catarina. Caminhos de Geografia, 8(17): 72-84.

MCTI - MINISTÉRIO DA CIÊNCIA, TECNOLOGIA E INOVAÇÃO. 2013. Vulnerabilidade das megacidades brasileiras às mudanças climáticas: tendências climáticas de longo prazo detectadas na Região Metropolitana de São Paulo. São Paulo: INPE. Disponível em http:// megacidades.ccst.inpe.br/sao_paulo/VRMSP/ capitulo4.php. Acessado em 18 abr. 2013. 
MILLER F.; BOWEN K. 2013. Questioning the assumptions: the role of vulnerability assessments in climate change adaptation. Impact Assessment and Project Appraisal, 31(3): 190-197.

PELOGGIA, A.U.G. 1998. O Homem e a Ambiente Geológico: geologia, sociedade e ocupação urbana no Município de São Paulo. Xamã, São Paulo, 271 p.

PELOGGIA, A.U.G. 2005. A cidade, as vertentes e as várzeas: a transformação do relevo pela ação do Homem no Município de São Paulo. Revista do Departamento de Geografia, 16: 24-31.

PELOGGIA,A.U.G.2011.Análisemultidimensional e gestão dos riscos geológicos: uma primeira aproximação. In: ABGE, CONGRESSO BRASILEIRO DE GEOLOGIA DE ENGENHARIA E AMBIENTAL, 13, São Paulo, Anais, 10 p. (CD-ROM).

PELOGGIA, A.U.G.; SILVA E.N.; NUNES, J.O.R. 2014a. Technogenic landforms: conceptual framework and application to geomorphologic mapping of artificial ground And landscape as transformed by human geological action. Quaternary and Environmental Geosciences, 5(2): 67-81.

PELOGGIA,A.U.G.; OLIVEIRA,A.M.S.; OLIVEIRA A.A.; SILVA E.N.; NUNES, J.O.R. 2014b. Technogenic geodiversity: a proposal on the classification of artificial ground. Quaternary and Environmental Geosciences, 5(1): 28-40.

PONCIANO, L. 2004. São Paulo: 450 bairros, 450 anos. Ed. Senac, São Paulo, 2 ed., 362 p.

RAMOS, C.S.; SANCHEZ M.C. 2000. Estudo metodológico de classificação de dados para cartografia temática. Geografia, 25(2): 23-52.
SILVA, C.H.R.T. 2012. Desastres naturais e desenvolvimento sustentável. Brasília: Senado Federal, Consultoria Legislativa. Disponível em https://www12.senado.leg.br/publicacoes/ estudos-legislativos/tipos-de-estudos/ outras-publicacoes/temas-e-agendas-parao-desenvolvimento-sustentavel/desastresnaturais-e-desenvolvimento-sustentavel. Acessado em 18 ago. 2016.

SUENAGA, N.I.T.; PUNTONI, A. 2010. Jardim Pantanal: Projeto Praia Parque. FAU/USP, São Paulo. Disponível em http://issuu.com/ niltonsuenaga/docs/tfg. Acessado em 10 mar. 2013.

TAYLOR, A.; BUTTERFIELD, R. 2011. Vulnerability definitions. We Adapt: Collaborating on Climate Adaptation. Disponível em http://weadapt.org/ knowledge-base/vulnerability/ vulnerabilitydefinitions. Acessado em 02 nov. 2012.

UN-ISDR - UNITED NATIONS INTERAGENCY SECRETARIAT OF THE INTERNATIONAL STRATEGY FOR DISASTER REDUCTION. 2002. Living with risk: a global review of disaster reduction initiatives. Preliminary version. ADRC - ISDR - WMO, Geneva, 384 p.

VILLA, F.; MCLEOD, H. 2002. Environmental vulnerability indicators for environmental planning and decision-making: guidelines and applications. Environmental Management, 29(3): 335-348.

WATTS, M.J.; BOHLE, H.G. 1993. The Space of Vulnerability: the causal structure of hunger and famine. Progress in Human Geography, 17(1): 43-67.

\section{Endereços dos autores:}

Camila Capassi Malagodi - Radboud University, Nijmegen School of Management, Thomas van Aquinostraat 3,6525 GD, Nimega, Holanda.E-mail: camila.capassi@gmail.com

Alex Ubiratan Goossens Peloggia - Universidade de Guarulhos, Centro de Pós-Graduação e Pesquisa, Praça Teresa Cristina 229, CEP 07023-070, Guarulhos, SP, Brasil.E-mail: alexpeloggia@uol.com.br 